\title{
Commentary on "Effect of green-Mediterranean diet on intrahepatic fat: the DIRECT PLUS randomised controlled trial"
}

\author{
Francesco Baratta, Daniele Pastori, Maria Del Ben \\ Department of Clinical Internal, Anesthesiological and Cardiovascular Sciences, Sapienza University of Rome, Rome, Italy \\ Correspondence to: Francesco Baratta. Department of Clinical Internal, Anesthesiological and Cardiovascular Sciences, Sapienza University of Rome, \\ Rome, Italy. Email: francesco.baratta@uniroma1.it. \\ Comment on: Yaskolka Meir A, Rinott E, Tsaban G, et al. Effect of green-Mediterranean diet on intrahepatic fat: the DIRECT PLUS randomised \\ controlled trial. Gut 2021. [Epub ahead of print]. doi: 10.1136/gutjnl-2020-323106.
}

Submitted Jul 09, 2021. Accepted for publication Aug 26, 2021.

doi: 10.21037/hbsn-21-280

View this article at: https://dx.doi.org/10.21037/hbsn-21-280

Non-alcoholic fatty liver disease (NAFLD) represents the most prevalent liver disease worldwide with a high spread in Western countries. This high spread is due to the typical Western lifestyles. In fact, western diet, characterized by the high intake of red meat, processed meat, high-fat dairy products and high-sugar drinks and sedentary lifestyles are the principal factors promoting the growing prevalence of obesity and NAFLD together (1).

In the recent study "Effect of green-Mediterranean diet on intrabepatic fat: the DIRECT PLUS randomised controlled trial" (2), Yaskolka Meir et al. investigated the impact of dietary strategies based on Mediterranean Diet (MedDiet) on NAFLD. Moreover, they also contributed to the discussion about factors associated with NAFLD pathogenesis and promoted a specific nutritional approach for NAFLD treatment.

In their randomized clinical trial, the Authors reported a median reduction of $19.6 \%$ in intrahepatic fat content (IHF) in patients who adopted MedDiet, reaching a median reduction of $38.9 \%$ in patients who took MedDiet enriched with plants and food rich in polyphenols and restricted in red meat (green-MedDiet). Of note, Authors observed a median IHF reduction of $12.2 \%$ also in patients enrolled in the control group who were trained with healthy diet guidelines (HDG). MedDiet and green-MedDiet induced higher weight loss in comparison to HDG (-2.7 vs. -3.7 vs. $-0.4 \mathrm{~kg}$, respectively). The differences in weight loss partially explains those in IFH reduction between MedDiet and HDG. This finding is not surprising as international guidelines for the management of fatty liver disease already reported that weight loss, beyond the strategies used to, is the best approach to reduce fatty liver accumulation (3). However, in the group of green-MedDiet, IHF reduction is independent from weight loss. This effect mainly depended on the restriction of red meat and on the amount of vegetables intake.

In addition, Authors investigated a possible effect of gut microbiota shift induced by MedDiet in the reduction of IHF. According to previous evidence (4), they found a reduction in Ruminococcaceae Familia at baseline and, after MedDiet, IFH changes correlated with the improvements of specific Ruminococcaceae Genera. Two specific bacteria, Fournierella and Ruminococcaceae_UCG-014 were found to be significantly affected by lifestyle intervention. In turn, the enrichment of these two bacteria resulting from 18 months of MedDiet correlated with IHF reduction.

In addition to gut microbiota improvement, Authors found a reduction in pro-inflammatory chemokines from different visceral tissues. These findings, together, put in lines most of the hypotheses previously assumed on the role of gut microbiota in NAFLD pathogenesis $(4,5)$. The concurrent enrichment of gut microbiota variety, the reduction in adipokines, hepatokines and inflammatory mediators and the decrease in IFH add new strong evidence on the tight association among dietary habits, gut microbiota and NAFLD. Unfortunately, Authors didn't explore the impact of MedDiet on gut permeability, a mechanism poorly investigated in the intricacies of the relationship between MedDiet and NAFLD. Leaky gut is a well-known mechanism related to NAFLD 
pathogenesis (4) and processes by wich dietary habits, via gut microbiota modulation, induce it is widley debated. Different mechanisms were investigated. Unsaturated fatty acids have been reported to be able to modulate intestinal barrier integrity; oxidized fatty acids induce the apoptosis of enterocytes; high-fat diets induce high bile acids secretion and bile acids stimulate the epidermal growth factor receptor (EGFR), leading to an impaired gut permeability (6). However, poor data demonstrated a link between leaky gut and the presence of high circulating levels of bacterial product in the blood of NAFLD patients who poorly adherer to MedDiet $(7,8)$.

Finally, in our opinion, authors poorly focused their attention on a key finding of their research. Yaskolka Meir, and collogues found a strong correlation between insulin resistance (IR) and IFH reduction. IR is a key mechanism in NAFLD pathogenesis and many authors consider NAFLD as a liver manifestation of the metabolic syndrome (9). In fact, previously observational and interventional studies $(7,10)$ demonstrated the interplay among MedDiet, IR and NAFLD. In addition, it would be very interesting to investigate the possible correlation between gut microbiota shift and IR improvement.

In conclusion, data from the DIRECT PLUS trial add new evidence on the favorable effect of MedDiet on NAFLD. Moreover, the accuracy of the study design and the wide number of the investigated variables contribute to better understand the multifactorial processes involved in NAFLD pathogenesis and the mechanisms by which MedDiet reduces IHF till NAFLD resolution.

To the date, DIRECT PLUS represents another brick in the wall of the evidence proving that MedDiet is the best nutritional approach for NAFLD management.

\section{Acknowledgments}

Funding: None.

\section{Footnote}

Provenance and Peer Review: This article was commissioned by the editorial office of Hepatobiliary Surgery and Nutrition. The article did not undergo external peer review.

Conflicts of Interest: All authors have completed the ICMJE uniform disclosure form (available at https://hbsn. amegroups.com/article/view/10.21037/hbsn-21-280/coif). The authors have no conflicts of interest to declare.
Ethical Statement: The authors are accountable for all aspects of the work in ensuring that questions related to the accuracy or integrity of any part of the work are appropriately investigated and resolved.

Open Access Statement: This is an Open Access article distributed in accordance with the Creative Commons Attribution-NonCommercial-NoDerivs 4.0 International License (CC BY-NC-ND 4.0), which permits the noncommercial replication and distribution of the article with the strict proviso that no changes or edits are made and the original work is properly cited (including links to both the formal publication through the relevant DOI and the license). See: https://creativecommons.org/licenses/by-nc-nd/4.0/.

\section{References}

1. Younossi Z, Anstee QM, Marietti M, et al. Global burden of NAFLD and NASH: trends, predictions, risk factors and prevention. Nat Rev Gastroenterol Hepatol 2018;15:11-20.

2. Yaskolka Meir A, Rinott E, Tsaban G, et al. Effect of green-Mediterranean diet on intrahepatic fat: the DIRECT PLUS randomised controlled trial. Gut 2021. [Epub ahead of print]. doi: 10.1136/ gutjnl-2020-323106.

3. European Association for the Study of Obesity (EASO). EASL-EASD-EASO Clinical Practice Guidelines for the management of non-alcoholic fatty liver disease. J Hepatol 2016;64:1388-402.

4. Plaza-Díaz J, Solís-Urra P, Rodríguez-Rodríguez F, et al. The Gut Barrier, Intestinal Microbiota, and Liver Disease: Molecular Mechanisms and Strategies to Manage. Int J Mol Sci 2020;21:8351.

5. Leung C, Rivera L, Furness JB, et al. The role of the gut microbiota in NAFLD. Nat Rev Gastroenterol Hepatol 2016;13:412-25.

6. Ferro D, Baratta F, Pastori D, et al. New Insights into the Pathogenesis of Non-Alcoholic Fatty Liver Disease: Gut-Derived Lipopolysaccharides and Oxidative Stress. Nutrients 2020;12:2762.

7. Baratta F, Pastori D, Polimeni L, et al. Adherence to Mediterranean Diet and Non-Alcoholic Fatty Liver Disease: Effect on Insulin Resistance. Am J Gastroenterol 2017;112:1832-9.

8. Baratta F, Pastori D, Bartimoccia S, et al. Poor Adherence to Mediterranean Diet and Serum Lipopolysaccharide are Associated with Oxidative Stress in Patients with Non- 
Alcoholic Fatty Liver Disease. Nutrients 2020;12:1732.

9. Takaki A, Kawai D, Yamamoto K. Multiple hits, including oxidative stress, as pathogenesis and treatment target in non-alcoholic steatohepatitis (NASH). Int J Mol Sci 2013;14:20704-28.

Cite this article as: Baratta F, Pastori D, Del Ben M. Commentary on "Effect of green-Mediterranean diet on intrahepatic fat: the DIRECT PLUS randomised controlled trial”. HepatoBiliary Surg Nutr 2021;10(5):699-701. doi: 10.21037/hbsn-21-280
10. Aller R, Sigüenza R, Pina M, et al. Insulin resistance is related with liver fibrosis in type 2 diabetic patients with non-alcoholic fatty liver disease proven biopsy and Mediterranean diet pattern as a protective factor. Endocrine 2020;68:557-63. 